\title{
Clinical expertise in the era of evidence-based medicine
}

\author{
and patient choice
}

\section{Scenario}

You are caring for a 68 year old man who has hypertension (intermittently controlled) with a remote gastrointestinal bleed and non-valvular atrial fibrillation (NVAF) for 3 months, and an enlarged left atrium (so cardioversion is unlikely). The patient has no history of stroke or transient ischaemic attack. His father experienced a debilitating stroke several years ago and when he learns that his atrial fibrillation places him at higher risk for a stroke, he is visibly distressed.

\section{Introduction}

The concepts of evidence-based medicine are evolving as limitations of early models are addressed. In this editorial, we present a new model for evidence-based clinical decision making based on patients' circumstances, patients' preferences and actions, and best research evidence, with a central role for clinical expertise to integrate these components.

\section{Evidence-based clinical practice in evolution}

Traditionally, clinicians have been credited with clinical acumen according to their skills in making a diagnosis and prescribing or administering a treatment. The advent of major investments in biomedical research, leading to new and better tests and treatments, has spurred the development of critical appraisal of the medical literature and evidence-based medicine, ${ }^{1}$ and application of current best evidence from healthcare research is now an expected adjunct to clinical acumen.

Initially, evidence-based medicine focused mainly on determining the best research evidence relevant to a clinical problem or decision and applying that evidence to resolve the issue. This early formulation de-emphasised traditional determinants of clinical decisions, including physiological rationale and individual clinical experience. Subsequent versions of evidencebased decision making have emphasised that research evidence alone is not an adequate guide to action. Rather, clinicians must apply their expertise to assess the patient's problem and must also incorporate the research evidence and the patient's preferences or values before making a management recommendation (figure 1). ${ }^{2}$

Figure 1 is based on the first text of evidence-based medicine ${ }^{3}$ and was published in an editorial that appeared in ACP Journal Club and Evidence-Based Medicine in 1996, along with this definition: "Evidence-based medicine is the conscientious and judicious use of current best evidence from clinical care research in the management of individual patients". ${ }^{4}$ The editorial also included the admonition that the definition of evidence-based medicine is evolving and will continue to be refined. Indeed, the concepts of evidence-based medicine have evolved considerably, and this model needs to be enhanced, especially for what is meant by clinical expertise and how it best fits in the overall picture of evidence-based medicine.

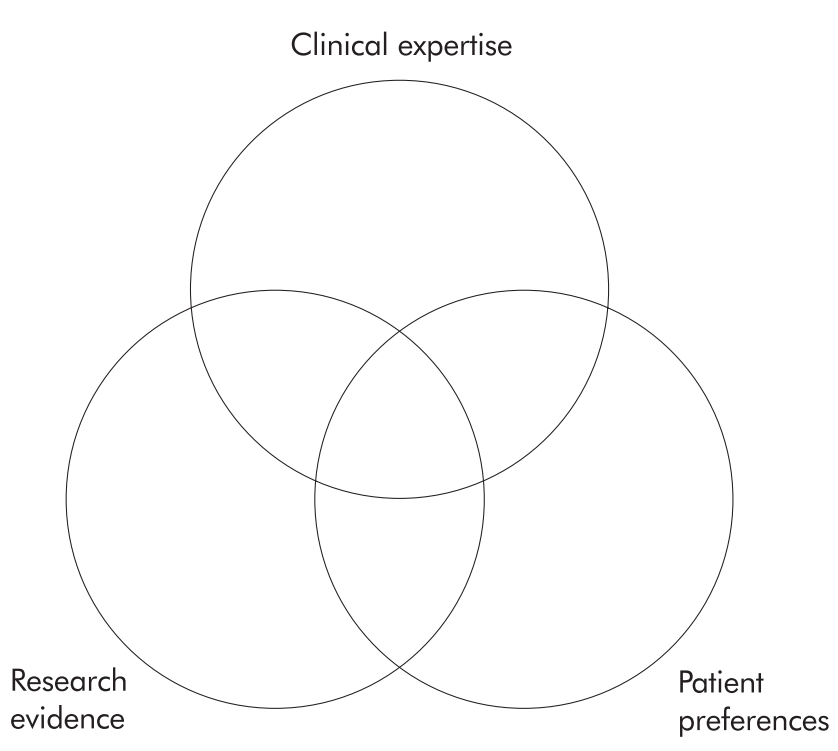

Figure 1 Early model of the key elements for evidence-based clinical decisions

\section{A new, prescriptive model for evidence-based} clinical decisions

Figure 2 depicts a more advanced model for evidence-based decisions, which have more recently been defined as "the integration of best research evidence with clinical expertise and patient values". This model is prescriptive rather than descriptive. That is, it is a guide for thinking about how decisions should be made rather than a schema for how they are made. For instance, at present, clinicians' individual preferences (as distinct from clinical expertise) often play a large role in their actions, leading to large practice variations in managing similar cases. When faced with critically ill patients with identical circumstances, different clinicians may, according to their preferences, institute aggressive life-prolonging interventions or withdraw life support. ${ }^{6}$ Our model acknowledges that patients' preferences rather than clinicians' preferences should be considered first whenever it is possible to do so.

In Figure 2, the "clinical state and circumstances" of the patient replace "clinical expertise" as one of the key elements in clinical decisions; "patient preferences" is expanded to include patients' actions and is reversed in position with "research evidence", which signifies its frequent precedence. Finally, "clinical expertise" is overlaid as the means to integrate the other 3 components; thus, constituting a 4th element. We will describe each of the components, their order, and the role of clinical expertise in integrating them. 


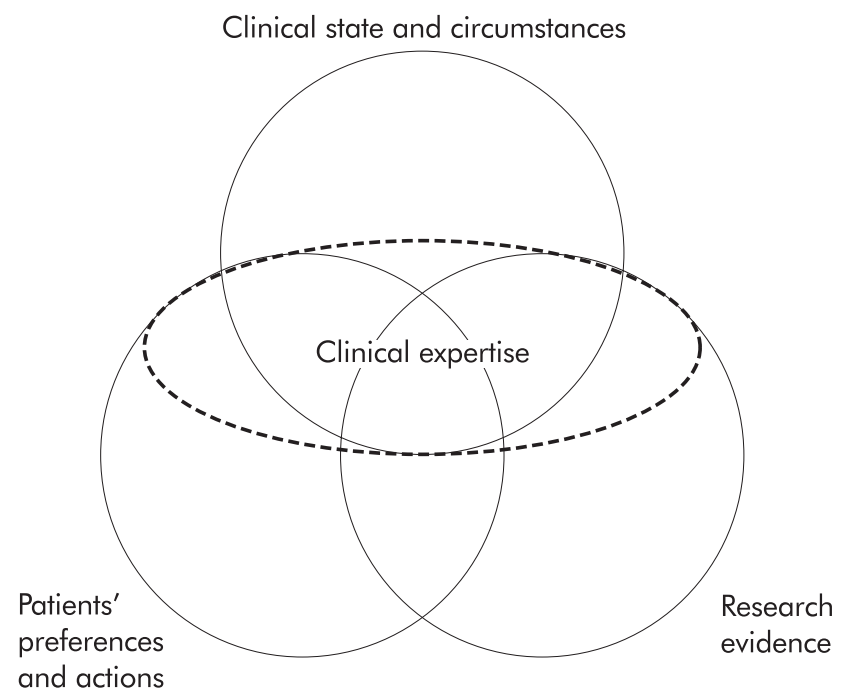

Figure 2 An updated model for evidence-based clinical decisions.

\section{Clinical state and circumstances}

Patients' clinical state, the clinical setting, and the clinical circumstances they find themselves in when they seek medical attention are key, and often dominant, factors in clinical decisions. For example, a patient with an undiagnosed symptom cannot be readily moved from a diagnostic decision to a therapeutic decision. Furthermore, people who find themselves in remote areas when beset by crushing retrosternal chest pain may have to settle for aspirin, whereas those living close to a tertiary care medical centre will probably have many more options - if they recognise the symptoms and act promptly! Similarly, a patient with atrial fibrillation and a high bleeding risk, as with the patient described at the beginning of this editorial, may experience more harm than good from anticoagulation treatment, whereas a patient with a high risk for stroke and a low risk for bleeding may have a substantial net benefit from such treatment. These states and circumstances can often be modified, for example, by improving the benefit: risk ratio by closer anticoagulant monitoring; thus, an "evidence-based" decision about anticoagulation for a patient with atrial fibrillation is not only determined by the proven efficacy of anticoagulation and its potential adverse effects, ${ }^{7}$ but it will also vary from patient to patient according to individual clinical circumstances.

\section{Patients' preferences and actions}

Patients may have either no views or unshakable views on their treatment options, depending on their condition, personal values and experiences, degree of aversion to risk, healthcare insurance and resources, family, willingness to take medicines, accurate or misleading information at hand, and so on. For our patient with NVAF, research evidence informs us about the differing preferences of patients and their physicians for antithrombotic treatment in atrial fibrillation when they weigh the competing risks for stroke and bleeding. ${ }^{8}$ On average, patients were willing to accept 17 extra major bleeding events in 100 patients over a 2 year period if warfarin prevented 8 strokes among these 100 patients; however, physicians were only willing to accept 10 major bleeding events for this benefit. Furthermore, considerable variability existed among both patients and physicians, suggesting that physicians would differ in their decisions concerning the same patient. This underscores the specification of the model that individual patient preferences must be taken into account.

Regardless of what their preferences may be, patients' actions may differ from both their preferences and their clinicians' advice. ${ }^{9}$ For example, a patient may prefer to lose weight, quit smoking, and take her medications as prescribed, but her actions may fall short of achieving any of these objectives. Alternatively, she may follow the treatment as prescribed, even if she resents its imposition, adverse effects, and costs. Unfortunately, clinicians' estimates of their patients' adherence to prescribed treatments have accuracy no better than chance. ${ }^{10}$ Thus, physicians' decisions for care will better meet the model's specifications if they are able to assess whether their patients will follow or are following their prescriptions. ${ }^{10}$

\section{Research evidence}

We title this component of clinical decisions "research evidence" to distinguish it from other forms of information that have always been part of clinical decisions, such as the patient's history, physical findings, diagnostic tests, circumstances, and stated preferences. Research evidence includes systematic observations from the laboratory, preliminary pathophysiological studies in humans, and more advanced applied clinical research, such as randomised controlled trials with outcomes that are immediately important to patients. Evidence-based medicine recognises that such evidence is not "created equal" and provides detailed guides for finding the most rigorous and pertinent evidence for a specific clinical decision. ${ }^{11}$

Because of the sustained investment in basic and applied health research, and the advances in applied health research methodology, an ever increasing amount of research evidence of direct relevance to patient care is available; however, much of the innovation is of marginal advantage at best, is often expensive, and often invokes risk for the patient even as it conveys benefit. Furthermore, an increasing number of alternatives with differing benefit and risk profiles exist for many conditions. For example, a systematic review of trials of anticoagulant and antiplatelet interventions for $\mathrm{NVAF}^{7}$ documents a $62 \%$ relative risk reduction for stroke from warfarin, offset by a $50 \%$ relative risk increase for major bleeding. Aspirin reduces the relative risk for stroke by $22 \%$, but without a statistically significant increase in the risk for bleeding.

These figures are averages derived from randomised controlled trials. Within these trials it is possible to identify subgroups of patients for whom the risk for stroke varies according to several factors, including age, history of hypertension, diabetes, and previous stroke or transient ischaemic attack. ${ }^{12}$ For the patient in our scenario, the risk for stroke in the first year after onset of NVAF would be about $6 \%$. The risk for bleeding while receiving warfarin also varies according to individual patient characteristics, including age, history of stroke and gastrointestinal bleeding, and presence of various comorbid conditions. ${ }^{13}$ For our patient, the 1 year risk for bleeding while receiving warfarin treatment would be about $8 \%$, which is higher than his untreated risk for stroke. "Personalising" the evidence to fit a specific patient's circumstances is a key area for development in evidence-based medicine. Notably, research evidence is often available to assist with the quantitative interpretation of the patient's clinical circumstances - in this case, the way in which the patient's hypertension and previous history of gastrointestinal bleeding affect his potential benefit and harm from anticoagulation and antiplatelet treatment.

\section{The expanded role of clinical expertise}

Clinical expertise includes the general basic skills of clinical practice as well as the experience of the individual practitioner. Clinical expertise must encompass and balance the patient's clinical state and circumstances, relevant research evidence, and the patient's preferences and actions if a successful and satisfying result is to occur. Accomplishing this goal often involves 
sorting through tradeoffs. The traditional turf of clinical expertise characterised by sizing up the patient's clinical state has never been more important; getting the diagnosis and prognosis right and knowing how to administer a treatment (whether just a pill or a complex technical procedure) often demand more skill now than ever before because the options are many and more is riding on doing things right. Clinicians must be atop not only the research evidence, but they must also acquire and hone the skills needed to both interpret the evidence and apply it appropriately to the circumstances - doing the right things. Finally, although communication with patients has always been important, determining the role in decision making that patients desire, ascertaining their preferences, and providing patients with the information they need to make an informed choice has never been more challenging. We know that patients often do not wish to be involved in making key decisions about their health; however, even when they do want to be involved, we do not know the ideal way to present information to patients. ${ }^{14}$ We do know that presenting information in relative terms (eg, warfarin will decrease your risk for having a stroke by $60 \%$ ) is problematic because patients assume their baseline risk is $100 \%$ even when clearly informed that their baseline risk is not $100 \%{ }^{15}$ Thus, determining what the patient wants and factoring this into the decision process is a growing responsibility of clinical expertise, currently limited by our understanding of how to determine patients' preferences.

\section{The varying role of the 4 components in individual clinical decisions}

The model can accommodate different weights for each component of the decision (which could be depicted visually by varying the sizes of the circles). In the "average" older patient with atrial fibrillation and 1 or 2 additional risk factors for stroke, but without excess risk for bleeding, the large relative risk reduction in stroke with warfarin may be the primary determinant of patient management. In a patient unwilling to have regular monitoring of anticoagulation status, patient preferences will dominate. It is undoubtedly true that the expertise of clinicians varies. For a patient living in a remote area, the circumstances - limited access to anticoagulant monitoring or care for complications circumstances - may dominate the clinical decision. For the patient in our opening scenario, several elements (the large relative and absolute risk reductions with warfarin, the particular clinical state that includes intermittent hypertension control, and the patient's preferences that include a strong aversion to stroke) will probably play major roles.

\section{Application to individual patients}

For our patient, the evidence could be (briefly) summarised as follows. If you take warfarin, 1 tablet a day, with weekly blood checks to guide the dose, your risk for stroke in the first year will decrease from $6 \%$ (6 in 100) to $2.3 \%$ (about 2 in 100). Half of strokes caused by atrial fibrillation will be "major", resulting in permanent disability, and half will be "minor", allowing the person to function independently. ${ }^{12}$ The anticoagulant will also increase your risk for major bleeding from 1\% to $8 \%$. If you take aspirin, 1 tablet a day, instead of warfarin, you will have no need for blood tests to monitor the dose concentration and your risk for stroke will decrease from $6 \%$ to $4.7 \%$, without an appreciable increase in your risk for major bleeding. By major bleeding, we mean the loss of at least 2 units of blood in 7 days or any life threatening bleeding. ${ }^{13}$

Finally, the clinician's work is obviously not completed after a balanced decision has been made. In our NVAF example, whatever the decision is, clinical expertise is needed for follow up and monitoring. Furthermore, given the difficulty that patients have in following the treatments we prescribe, evidence-based interventions to assess adherence ${ }^{10}$ and to assist patients in following the prescribed treatment ${ }^{16}$ are needed.

\section{Some limitations of the proposed model}

Our model does not depict all of the elements involved. For example, we have not included the important roles that society and healthcare organisations play in providing and limiting resources for health services. Rather, our focus has purposely been on the decisions made by patients and their immediate healthcare providers, leaving the bigger picture for another discussion. It is also impossible to implement the model as prescribed. For example, at present, it is not possible to make an accurate prediction of the patient's likelihood of following a treatment programme of anticoagulation and monitoring. Thus, our model is conceptual rather than practical and remains under development. Its intent is to augment models for evidence-based clinical decisions that have been proposed to date, especially with respect to the role of clinical expertise and the emphasis to be placed on patients' preferences and actions and to stimulate discussion for the next evolution of evidencebased care.

\section{Conclusions}

As we continue our journey through the era of researchinformed health care, the benefits that our patients will receive and our satisfaction with our own clinical performance will depend increasingly on making care decisions that incorporate the clinical state and circumstances of each patient, their preferences and actions, and the best current evidence from research that pertains to the patient's problem. The nature and scope of clinical expertise must expand to balance and integrate these factors, dealing with not only the traditional focus of assessing the patient's state but also the pertinent research evidence and the patient's preferences and actions before recommending a course of action.

R BRIAN HAYNES, MD, PhD
P J DEVEREAUX, MD
GORDON H GUYATT, MD, MSc
McMaster University
Hamilton, Ontario, Canada

1 Evidence-based Medicine Working Group. JAMA 1992;268:2420-5.

2 Haynes RB, Sackett DL, Gray JR, et al. ACP J Club 1996;125:A14-6. Evidence-Based Medicine 1996;1:196-8.

3 Sackett DL, Richardson WS, Rosenberg W, et al. Evidence-based medicine: how to practice and teach EBM. Edinburgh: Churchill Livingstone, 1997.

4 Sackett DL, Rosenberg WM, Gray JA, et al. BMJ 1996;312:71-2.

5 Sackett DL, Straus SE, Richardson WS, et al. Evidence-based medicine: how to practice and teach EBM. 2d ed. Edinburgh: Churchill Livingstone, 2000.

6 Cook DJ, Guyatt GH, Jaeschke R, et al.JAMA 1995;273:703-8.

7 Hart RG, Benavente O, McBride R, et al. Ann Intern Med 1999;131:492501.

8 Devereaux PJ, Anderson DR, Gardner MJ, et al. BMJ 2001;323:1218-22.

9 Haynes RB. Improving patient adherence: state of the art, with a special focus on medication taking for cardiovascular disorders. In: Burke LE, Okene IS, editors. Patient compliance in healthcare and research. American Heart Association monograph series. Armonk, NY: Futura Publishing Co, 2001:3-21.

10 Stephenson BJ, Rowe BH, Haynes RB, et al.JAMA 1993;269:2779-81.

11 Guyatt G, Rennie D. Introduction: The Philosophy of Evidence-Based Medicine. In: Guyatt G, Rennie D, editors. Users'guides to the medical literature: a manual for Evidence-Based Clinical Practice. Chicago: American Medical Association, 2001:3-12.

12 Risk factors for stroke and efficacy of antithrombotic therapy in atrial fibrillation. Analysis of pooled data from five randomized controlled trials. Arch Intern Med 1994;154:1449-57.

13 Beyth RJ, Quinn LM, Landefeld CS. Am J Med 1998;105:91-4

14 Deber RB, Kraetschmer N, Irvine J. Arch Intern Med 1996;156:1414-20.

15 Malenka DJ, Baron JA, Johansen S, et al.J Gen Intern Med 1993;8:543-8.

16 Haynes RB, Montague P, Oliver T, et al. Cochrane Database Syst Rev 2001;(4):CD000011. 